\title{
Evaluation of quality of life after Functional Endoscopic Sinus Surgery (FESS) in chronic rhinosinusitis patients in Menoufia Governorate
}

\author{
Original \\ Essam Abdelwanees Behiry', Hewaida Mohamed Anwer Elshazly', Ibrahim Ahmed Abdel- \\ Article \\ Shafy', Hosam Adel Hussein ${ }^{1}$, Mustafa Ahmed Kadah ${ }^{3}$ \\ ${ }^{1}$ Department of Otorhinolaryngology, ${ }^{2}$ Department of Public Health and Community \\ Medicine, Faculty of Medicine, Menoufia University, ${ }^{3}$ Department of Otorhinolaryngology, \\ Shebin El-Kom Teaching Hospital, Egypt.
}

\begin{abstract}
Background: Chronic rhinosinusitis has a great impact on the quality of life. Functional endoscopic sinus surgery is the treatment of choice for chronic rhinosinusitis refractory to medical treatment as it allows restoring ventilation and mucociliary clearance. Sino- Nasal Outcome Test 22 questionnaire has been used for evaluating changes in symptoms and predicting the extent of postoperative outcome.

Objective: The aim of the study was to evaluate how functional endoscopic sinus surgery affects the outcome in patients' symptom profile and quality of life.

Patients and Methods: 60 patients indicated for functional endoscopic sinus surgery from March 2017 till December 2018. This prospective study was carried out at the Otorhinolaryngology Department at El-Menoufia University Hospital. For all patients, the Sino-Nasal Outcome Test-22 was completed before and three months after Functional Endoscopic sinus surgery.

Results: 60 patients were enrolled in this study (34 Males and 26 Females) aged from 25 to 40 years old with a mean age of $32.9 \pm 5.3$. The results of the statistical analysis showed that functional endoscopic sinus surgery reduced the SinoNasal Outcome Test-22 questionnaire score with a statistically high significant result $(P \leq 0.001)$.

Conclusion: Functional endoscopic sinus surgery statistical significant improvements in disease-specific quality of life for patients with chronic rhinosinusitis.
\end{abstract}

Key Words: Chronic rhinosinusitis, endoscopic sinus surgery, quality of life, SNOT-22

Received: 25 April 2019, Accepted: 08 July 2019

Corresponding Author: Mustafa Ahmed Kadah, Department of Otorhinolaryngology, Shebin El-Kom Teaching Hospital, Egypt, Tel.: +201003493235, E-mail: kadahmustafa@gmail.com

ISSN: 2090-0740, November 2019 Vol.20, No.3

\section{INTRODUCTION}

Functional endoscopic sinus surgery (FESS) is the most commonly used surgical technique to treat medically unresponsive chronic sinusitis and other serious conditions of the nasal sinuses that result in impaired sinus drainage with an improvement in symptoms of up to 90 percent may be expected as it removes obstructing tissues, allowing the sinuses to drain more naturally. This decrease the severity, frequency, and duration of infections ${ }^{[1]}$.

In order to evaluate the success rate of FESS, an assortment of variable outcome measures exist. One of the principal results of the evaluation is quality of life. The evaluation of patients' health-related quality of life shows a significant role in understanding patients' disease and treatment outcomes. They show how every patient feels about his symptoms, help to personalize the disease with better understand the patients' condition and his expectations with regard to treatment outcomes, and allows the physician to understand how a disease intervenes in everyday life of the patient, and thus improves patient/ doctor relationship and treatment outcome ${ }^{[2]}$.

There are many validated tools to evaluate the effect of nasal/sinus complaints upon QoL. Tools used to evaluate the quality of life are either general (generic) health questionnaires for evaluating general conditions or disease-specific questionnaires focused on symptoms of disease $\mathrm{e}^{[3]}$.

\section{PATIENTS AND METHODS}

This is a prospective Cohort clinical trial study carried out from March 2017 to December 2018 at the Otorhinolaryngology (ORL) Department in Menoufia University Hospital after the approval of the ethical committee of the hospital. All patients signed informed consent; the consent form was developed according to the 
stander of Quality Improvement System in the Ministry of Health in Egypt.

Our 60 patients included in our study had chronic rhinosinusitis (CRS) with or without nasal polyps that had not improved after three months of drug therapy and referred to FESS, patients aged from 25 years old to 40 years old, good overall health status and no systemic or localized diseases that might compromise the patient's health.

Exclusion criteria were patients who had previous endoscopic sinus surgery and patients with Psychic illness.

A full history was taken from all patients. General examination was done, followed by head and neck examination, a full nasal and nasopharyngeal examination that completed in the office. Anterior rhinoscopy using a headlight or mirror plus nasal speculum performed prior to any attempts of nasal decongestion. Any evidence of mucopurulent discharge, nasal polyps, enlarged turbinates, nasal septal deviation, or diseased mucosa was noted.

Using rigid naso-endoscopy, decongestion, and anesthesia with a sprayed mixture of $4 \%$ lidocaine with phenylephrine to facilitate the examination. Computed topography was done for all patients preoperatively. Routine pre-operative investigations: (Complete Blood Counts, Echocardiography, bleeding profile, liver function, kidney function) were done for all patients. All patients fulfilled the Sinonasal Outcome Test22 (SNOT22) at the day before the operation.

The surgical procedures were performed along with the guidelines described by Messerklinger and Stammberger ${ }^{[4]}$. The extent of surgery was determined by the severity of disease and the extent of involvement of sinuses as preoperative CT scan and nasal endoscopy. It consisted of uncinectomy, middle meatal antrostomy, anterior ethmoidectomy, posterior ethmoidectomy, sphenoidotomy, frontal sinus procedures, with or without septoplasty and inferior turbinate reduction. Patients underwent functional endoscopic sinus surgery under general anesthesia using rigid naso-endoscopy (zero, 30 and 70-degree $17 \mathrm{~cm}$ $4 \mathrm{~mm}$ endoscopy Karl Storz, Germany) and endoscopic equipment with standard instrumentation. Navigationassisted surgery was not available in our department.

During postoperative care, all 60 cases were hospitalized for 48 hours then were discharged after removal of anterior nasal packing with routine precautions of prophylactic systemic antibiotics (amoxicillinclavulanic acid $1 \mathrm{gm} / 12 \mathrm{~h}$ ) orally for 14 days, analgesics (acetaminophen(paracetamol)500 mg / 6h maximum 4gm) orally for 10 days, topical steroids, budesonide spray 64 mcg on each nostril every $12 \mathrm{~h}$ started 15 days after surgery and continued if necessary. Patients also used alkaline nasal douche solution $20 \mathrm{ml}$ on each nostril every 6 hours started just after removal of anterior nasal packing until the surgical wound was completely healed and no crust was seen in the nasal cavity under endoscopic examination. Follow-up visits were done weekly for the first month then every month. In each visit nasal suctioning was done, crusts were removed and nasal cavity re-examined using rigid nasoendoscopic examination to exclude complications as nasal adhesions. All patients fulfilled the SNOT22 after 3 months postoperatively.

\section{Statistical analysis}

Data were collected, tabulated, statistically analyzed using a personal computer with Statistical Package of Social Science (SPSS) version 22 and Epi Info 2000 programs, where the following statistics were applied. Two types of statistics were done: Descriptive statistics e.g. Number (No), percentage (\%), mean $\left(\mathrm{X}^{-}\right)$and standard deviation (SD), and Analytic statistics: Mann-Whitney test and Wilcoxon test. (P-value $>0.05$ to be statistically insignificant, $P$-value $\leq 0.05$ to be statistically significant and $P$-value $\leq 0.001$ to be highly statistically significant)

\section{RESULTS}

A total of 60 patients included in this study aged from 25 to 40 years old with a mean age of $32.9 \pm 5.3$. As shown in (Table 1), there were 34 males and 26 females included in our study. Study participants were asked to complete the SNOT-22 that were compared preoperatively and 3 months postoperatively. The 22-items of the SNOT-22 were categorized into 5 symptom domain scores: rhinologic symptoms domain; extranasal rhinologic symptoms domain; ear/facial symptoms domain; psychological dysfunction domain; and sleep dysfunction domain.

Table 1: Demographics data and diagnosis of studied patients (No. $=60)$

\begin{tabular}{lcc}
\hline & \multicolumn{2}{c}{ Studied patients (No.=60) } \\
\hline Age (Years) & \multicolumn{2}{c}{$32.9 \pm 5.3$} \\
Mean \pm SD & No & $\%$ \\
\cline { 2 - 3 } Gender & & \\
Male & 34 & 56.7 \\
Female & 26 & 43.3 \\
Diagnosis & & \\
CRSwNP & 42 & 70 \\
CRSsNP & 18 & 30 \\
\hline CRSwNP=Chronic Rhinosinusitis with nasal polyps \\
CRSsNp=Chronic Rhinosinusitis without nasal polyps
\end{tabular}

Regarding domain of SNOT -22 questionnaire comparison between cases pre and postoperative results, there was a significant high difference in all domains of SNOT22 questionnaire pre and postoperative results (rhinologic symptoms domain, extranasal rhinologic symptoms domain, ear/facial symptoms domain, psychological dysfunction domain and sleep dysfunction domain). (Table 2) 
Table 2: Comparison between cases pre and post-operative results as regard domain of SNOT -22 questionnaire

\begin{tabular}{|c|c|c|c|c|}
\hline \multirow{3}{*}{ Domains of SNOT questionnaire } & Pre-operative & Post-operative & \multirow{3}{*}{$\mathrm{W}$} & \multirow{3}{*}{$P$} \\
\hline & & & & \\
\hline & Mean \pm SD & Mean \pm SD & & \\
\hline Rhinologic Symptoms Domain & $19 \pm 4.9$ & $5.1 \pm 3.3$ & 6.74 & $<0.001$ \\
\hline Extra-Nasal Rhinologic Symptoms Domain. & $5 \pm 1.9$ & $1.16 \pm 1.16$ & 6.7 & $<0.001$ \\
\hline Ear/Facial Symptoms Domain. & $8.40 \pm 4.37$ & $1.88 \pm 2.77$ & 6.5 & $<0.001$ \\
\hline Psychological Dysfunction Domain & $14.91 \pm 7.53$ & $2.38 \pm 4.46$ & 6.3 & $<0.001$ \\
\hline Sleep Dysfunction Domain & $8.25 \pm 3.49$ & $1.11 \pm 2.76$ & 6.42 & $<0.001$ \\
\hline
\end{tabular}

W=Wilcoxon Signed Ranks Test

$P$ value: $\mathrm{NS}=$ Non-significant $(P$-value $>0.05), \mathrm{S}=\operatorname{significant}(P$-value $\leq 0.05 \mathrm{HS}=$ highly significant $(P$-value $\leq 0.001)$.

Regarding the mean scores of SNOT -22 questionnaire domains comparison between males and females patients postoperatively, there was no significant difference between males and females outcome. (Table 3)

Table 3: Comparison between males and females patients post-operative outcome regarding the mean scores of SNOT -22 questionnaire domains

\begin{tabular}{|c|c|c|c|c|}
\hline \multirow{2}{*}{ Domains of SNOT questionnaire } & Male No. $=34$ & Female No. $=26$ & \multirow{2}{*}{$\begin{array}{c}\text { Maan- } \\
\text { whiteny test }\end{array}$} & \multirow{2}{*}{$P$} \\
\hline & Mean \pm SD & Mean \pm SD & & \\
\hline Rhinologic Symptoms Domain & $5.11 \pm 3.01$ & $5.23 \pm 3.86$ & 0.105 & 0.916 \\
\hline Extra-Nasal Rhinologic Symptoms Domain. & $1.14 \pm 1.28$ & $1.19 \pm 1.01$ & 0.54 & 0.57 \\
\hline Ear/Facial Symptoms Domain. & $1.94 \pm 42.60$ & $1.80 \pm 3.03$ & 0.30 & 0.76 \\
\hline Psychological Dysfunction Domain & $2.23 \pm 3.64$ & $2.57 \pm 5.4$ & 0.124 & 0.90 \\
\hline Sleep Dysfunction Domain & $1.17 \pm 2.72$ & $1.03 \pm 2.8$ & 0.46 & 0.64 \\
\hline
\end{tabular}

$\mathrm{W}=$ Wilcoxon Signed Ranks Test

$P$ value: $\mathrm{NS}=$ Non-significant $(P$-value $>0.05), \mathrm{S}=$ significant $(P$-value $\leq 0.05 \mathrm{HS}=$ highly significant $(P$-value $\leq 0.001)$.

According to diagnosis as regarding the mean scores of SNOT -22 questionnaire domains comparison between patients' postoperative results, there was a significant difference between CRSsNP and CRSwNP in rhinologic
Symptoms and extra-nasal rhinologic Symptoms postoperatively, where a decrease of the score in CRSwNP more than CRSsNP indicated better improvement but no difference in other domains results. (Table 4) 
Table 4: Comparison between patients post-operative results according diagnosis regarding the mean scores of SNOT -22 questionnaire domains

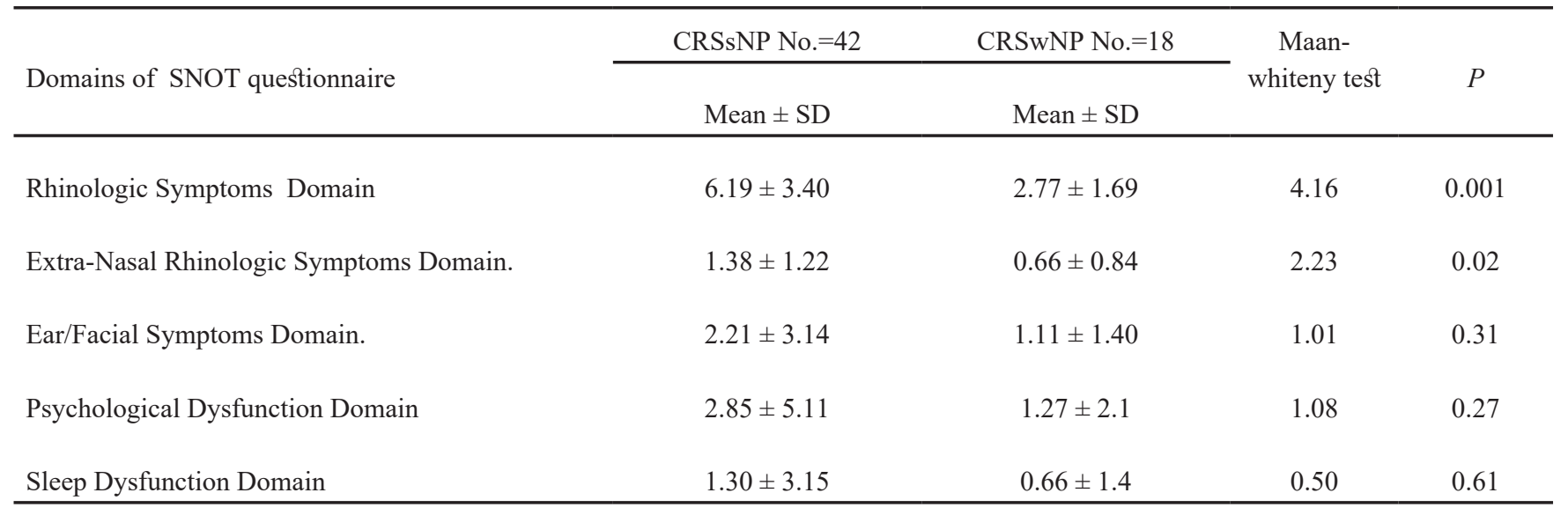

$\mathrm{W}=$ Wilcoxon Signed Ranks Test

$P$ value: NS $=$ Non-significant $(P$-value $>0.05), \mathrm{S}=$ significant $(P$-value $\leq 0.05 \mathrm{HS}=$ highly significant $(P$-value $\leq 0.001)$.

\section{DISCUSSION}

Clinical evaluation of chronic rhinosinusitis using major and minor criteria as well as endoscopy and CT-scan are common methods for the diagnosis and handling of the patients. Several studies have shown that the inflammatory degree shown in endoscopic findings and CT-scan are not directly related to the extent of the symptoms experienced by the patient. In that, although endoscopy and CT-scan show slight changes, the patients may have a serious complaint. On the other hand, sometimes the changes are, although large, the patients do not have many complaints. Due to uncertainties in diagnostic criteria and the lack of association between the imaging and symptoms of the disease, the quality of life Inventory is today the strongest evidence for evaluating the response to treatment ${ }^{[5]}$.

In the study, we used SNOT-22 which is a specific questionnaire to analyze the quality of life in sinonasal diseases adapted and validated in several languages and is gaining popularity in other rhinological conditions and procedures $^{[6]}$.

SNOT-22 stems from the SNOT-20. The scoring has been simplified by removing the importance rating and adding two items: nasal blockage and loss of sense of taste and smell. It evaluates nasal, paranasal and psychological symptoms and those associated with sleep. It has 22 questions graded from 0 to 5 ; 0 meaning no problems and 5 is the worst possible problem. The total sum of the questionnaire score 110 numerically indicates the effect of the disease in the QoL of the individual[ ${ }^{[7]}$.

Regarding the mean scores of SNOT-22 questionnaire domains comparison between males and females patients' postoperative results. In our study, there was no significant difference in improvement in the questionnaire score between males and females, the result of our study was in agreement with a randomized controlled trial study in Iran by Amalia et al. and a prospective cohort study in the United States by Adappa et al..$^{[8,9]}$. Also, another retrospective cohort study in the United States by Lal et al. and a prospective study in Brazil by Bezerra et al.$^{[10,11]}$ showed agreement with our results in this comparison.

Otherwise, a prospective study in Nova Scotians Canada by Macdonald et al. ${ }^{[12]}$, studied 26 patients that followed for 3 and 12 months postoperatively and concluded greater improvement in the quality of life was reported within males and those below 50 years, after FESS.

Regarding the impact of sinus surgery on sleep outcomes, in our study FESS improved sleep outcomes. Symptoms of excessive sleepiness and daytime fatigue are frequent complaints of patients with CRS. Because the effects of CRS are not only local but also systemic and that furthermore, FESS improves systemic cytokines levels by decreasing disease burden, it makes sense that FESS correspondingly improve sleep outcomes.

Result of our study was in agreement with a study in Canada done by Rotenberg et al. ${ }^{[13]}$, and another prospective multisite cohort study in the United States with a populationbased sample of 405 adults by Alt et al., ${ }^{[14]}$ concluded that patients with CRS have a high prevalence of sleep dysfunction that significantly improved following FESS.

Regarding pre and postoperative improvement as regard domain of SNOT -22 questionnaire, our study showed that all items of the questionnaire were highly significantly improved. Functional endoscopic sinus surgery is a minimally invasive technique used to restore sinus ventilation and normal function. It is the treatment of choice for CRS patients not responding to drug therapy 
as it removes obstructing tissues, allowing the sinuses to drain more naturally.

The result of our study was in agreement with a prospective cohort study performed in Morrocco by Laababsi et al., ${ }^{[15]}$ showed that FESS improves all domains of QoL. In a prospective cohort study in Finland by Alakärppä et al., ${ }^{[16]}$ showed also improvement in SNOT22 score after both septoplasty and endoscopic sinus surgery. In a retrospective analysis of prospectively collected patient data at the University of Virginia in the United States by Kennedy et al., ${ }^{[17]}$ showed that with optimal surgical intervention (and postoperative medical management), FESS is an extremely effective treatment of CRS. Patient-based outcome measures, such as the SNOT22, are helpful tools for quantifying changes in symptoms and, can be used to evaluate the extent of postoperative improvement. In a prospective cohort study in England and Wales by Hopkins et al., ${ }^{[18]}$ evaluate the effect of surgical treatment of CRS (with or without nasal polyp) on QoL using SNOT-22, 3128 patients with CRS were evaluated, confirmed significant improvement in the SNOT-22 score at 3,12 , and 36 months after surgery.

Regarding patients' postoperative improvement according to diagnosis, our study showed greater SNOT22 improvement in those with polyps more than without polyps. Our results were in agreement with a prospective study in Brazil by Kosugi et al, a prospective study in Iran by Saedi et al, and another retrospective study in the United States by Zhang et al ${ }^{[19,20,21]}$.

Our results were in disagreement with study in Brazil by Mascarenhas et al., ${ }^{[22]}$ that comprised of 38 patients with no difference in SNOT-22 improvement between polyp and non-polyp patients.

Many studies have shown the impact of functional endoscopic sinus surgery on QoL using disease-specific questionnaires, but to this date, there had been no studies of this sort done in El- Menoufia University hospital.

\section{CONCLUSION}

The outcome of chronic rhinosinusitis has significantly improved after functional endoscopic sinus surgery with better results regarding patients' local nasal, extranasal, psychological, ear/facial, and sleep dysfunction manifestations.

\section{CONFLICT OF INTERESTS}

There are no conflicts of interest

\section{REFERENCES}

1. Weber RK, Hosemann W. Comprehensive review on endonasal endoscopic sinus surgery. GMS current topics in otorhinolaryngology, head and neck surgery. 2015;14.
2. Nair S, Dutta A, Rajagopalan R, Nambiar S. Endoscopic sinus surgery in chronic rhinosinusitis and nasal polyposis: a comparative study. Indian Journal of Otolaryngology and Head \& Neck Surgery 2011 Jan; 63(1):50-5.

3. Schalek P. Rhinosinusitis-Its impact on the quality of life. In Peculiar Aspects of Rhinosinusitis 2011 Nov 23. IntechOpen.

4. Bhattacharyya N. Computed tomographic staging and the fate of the dependent sinuses in revision endoscopic sinus surgery. Archives of Otolaryngology-Head \& Neck Surgery 1999 Sep; 125(9):994-9.

5. Rosenfeld RM, Andes D, Bhattacharyya N, Cheung D, Eisenberg S, Ganiats TG, Gelzer A, Hamilos D, Haydon III RC, Hudgins PA, Jones S. Clinical practice guideline: adult sinusitis. OtolaryngologyHead and Neck Surgery. 2007 Sep 1;137(3): S1-31.

6. Elwany S, Atef A, Ibrahim AA, Ismail AS, Hussein WK, Youssef AS, et al. Arabic translation and validation of SNOT-22. The Egyptian Journal of Otolaryngology 2017 Oct; 33(4):611.

7. Poetker DM, LSmith T. Measuring Quality of Life and Outcomes in Rhinology. Sataloff's Comprehensive Textbook of Otolaryngology: Head \& Neck Surgery: Rhinology/Allergy and Immunology. 2015 Nov 30;2:169.

8. Amali A, Saedi B, Rahavi-Ezabadi S, Ghazavi $\mathrm{H}$, Hassanpoor N. Long-term postoperative azithromycin in patients with chronic rhinosinusitis: A randomized clinical trial. American journal of rhinology \& allergy 2015 Nov; 29(6):421-4.

9. Adappa ND, Farquhar D, Palmer JN, Kennedy DW, Doghramji L, Morris SA, et al. TAS2R38 genotype predicts surgical outcome in nonpolypoid chronic rhinosinusitis. InInternational forum of allergy \& rhinology 2016 Jan; 6(1):25-33.

10. Lal D, Golisch KB, Elwell ZA, Divekar RD, Rank MA, Chang YH. Gender-specific analysis of outcomes from endoscopic sinus surgery for chronic rhinosinusitis. InInternational forum of allergy \& rhinology 2016 Sep; 6(9):896-905.

11. Bezerra TF, Piccirillo JF, Fornazieri MA, Pilan RR, Pinna FD, Padua FG, et al. Assessment of quality of life after endoscopic sinus surgery for chronic rhinosinusitis. Brazilian journal of otorhinolaryngology 2012 Apr; 78(2):96-102.

12. Macdonald KI, McNally JD, Massoud E. Quality of life and impact of surgery on patients with chronic rhinosinusitis. Journal of Otolaryngology-Head \& Neck Surgery. 2009 Apr; 38(2). 
13. Rotenberg BW, Pang KP. The impact of sinus surgery on sleep outcomes. InInternational forum of allergy \& rhinology 2015 Apr; 5(4):329-332.

14. Alt JA, DeConde AS, Mace JC, Steele TO, Orlandi RR, Smith TL. Quality of life in patients with chronic rhinosinusitis and sleep dysfunction undergoing endoscopic sinus surgery: a pilot investigation of comorbid obstructive sleep apnea. JAMA otolaryngology-head \& neck surgery. 2015 Oct; 141(10):873-81.

15. Laababsi R, Elkrimi Z, Allouane A, Rouadi S, Abada $\mathrm{R}$, Roubal M, et al. Quality of life outcomes of patients with chronic rhinosinusitis after functional endoscopic sinus surgery, prospective cohort study. Annals of Medicine and Surgery. 2019 Mar; 10.

16. Alakärppä AI, Koskenkorva TJ, Koivunen PT, Alho OP. Predictive factors of a beneficial quality of life outcome in patients undergoing primary sinonasal surgery: a population-based prospective cohort study. European Archives of Oto-Rhino-Laryngology. 2018 May; 275(5):1139-47.

17. Kennedy JL, Hubbard MA, Huyett P, Patrie JT, Borish L, Payne SC. Sino-nasal outcome test (SNOT-22): a predictor of postsurgical improvement in patients with chronic sinusitis. Annals of Allergy, Asthma \& Immunology. 2013 Oct; 111(4):246-51.
18. Hopkins C, Browne JP, Slack R, Lund V, Topham $\mathrm{J}$, Reeves B, et al. The national comparative audit of surgery for nasal polyposis and chronic rhinosinusitis. Clinical Otolaryngology. 2006 Oct; 31(5):390-8.

19. Kosugi EM, Chen VG, da Fonseca VM, Cursino MM, Neto JA, Gregório LC. Translation, crosscultural adaptation, and validation of SinoNasal Outcome Test (SNOT)-22 to Brazilian Portuguese. Brazilian journal of otorhinolaryngology. 2011 Sep; 77(5):663-9.

20. Saedi B, Sadeghi M, Akhavan-Khaleghi N, Seifmanesh H. Impact of endoscopic sinus surgery on the quality of life of patients with nasal polyposis. B-ENT. 2014; 10(1):59-65.

21. Zhang Z, Adappa ND, Doghramji LJ, Chiu AG, Lautenbach E, Cohen NA, et al. Quality of life improvement from sinus surgery in chronic rhinosinusitis patients with asthma and nasal polyps. InInternational forum of allergy \& rhinology 2014 Nov; 4(11):885-892.

22. Mascarenhas JG, Fonseca VM, Chen VG, Itamoto $\mathrm{CH}$, Silva CA, Gregório LC, et al. Long-term outcomes of endoscopic sinus surgery for chronic rhinosinusitis with and without nasal polyps Brazilian journal of otorhinolaryngology. 2013 Jun; 79(3):306-11. 